\title{
A parametric study of bridge load effect under stochastic vehicular load
}

\author{
He-Qing Mu \\ School of Civil Engineering and Transportation, \\ State Key Laboratory of Subtropical Building Science, \\ South China University of Technology, \\ Guangzhou 510640, China \\ Email: cthqmu@scut.edu.cn
}

\section{Hou-Zuo Guo and Tian-Yu Zhang}

School of Civil Engineering and Transportation, South China University of Technology, Guangzhou 510640, China

Email: ctguo.houzuo@mail.scut.edu.cn

Email:201536192683@mail.scut.edu.cn

\section{Cheng Su*}

School of Civil Engineering and Transportation,

State Key Laboratory of Subtropical Building Science, South China University of Technology, Guangzhou 510640, China

Email: cvchsu@scut.edu.cn

\begin{abstract}
Bridge vehicular load effect inference is one of the key factors in bridge reliability and life-cycle assessment. The stochastic vehicular load can be decomposed into two parts: 1) the random vehicular inflow, describing the information about the properties of the inflow vehicles entering the bridge; 2) the stochastic vehicular flow, describing the information about the vehicle-following pattern of vehicles on the bridge. In order to investigate how the bridge load effect is affected by the stochastic vehicular load, two key parameters of the stochastic vehicular load are selected: 1) the probability of the existence of vehicle $(\mathrm{PoV})$, controlling the traffic volume of the vehicular inflow entering the bridge; 2 ) the probability of random slowing down (PoSD), controlling the vehicle-following pattern of the stochastic vehicular flow. With different values of the PoV and the PoSD, the samples of the vehicular load effects are achieved by embedding the samples of the stochastic vehicular load and the influence lines of the load effects. The Gaussian process regression (GPR) is utilised to obtain the relations between two parameters of the stochastic vehicular load (the PoV and the PoSD) and the statistical moments [the mean and the standard deviation (STD)] of the simulated load effect samples. It turns out that the load effect is interactively influenced by the PoV and the PoSD.
\end{abstract}

Keywords: bridge vehicular load effect; Gaussian process regression; GPR; Nagel-Schreckenberg model; uncertainty. 
Reference to this paper should be made as follows: $\mathrm{Mu}, \mathrm{H}-\mathrm{Q}$., Guo, H-Z., Zhang, T-Y. and Su, C. (2019) 'A parametric study of bridge load effect under stochastic vehicular load', Int. J. Lifecycle Performance Engineering, Vol. 3, No. 1, pp.77-90.

Biographical notes: He-Qing $\mathrm{Mu}$ received his $\mathrm{PhD}$ in Civil Engineering from University of Macau, Macao, China. He was a visiting scholar of California Institute of Technology (Caltech) and University of California, Berkeley (UC Berkeley). $\mathrm{He}$ is currently an Associate Professor of Civil Engineering Department of South China University of Technology. His research interests include Bayesian inference, earthquake engineering, robust data analysis, structural health monitoring.

Hou-Zuo Guo received his BS degree in Civil Engineering from South China University of Technology, Guangzhou, China, in 2018. He is currently a Master student of South China University of Technology. His research interests include Bayesian inference, random vibration and uncertainty quantification.

Tian-Yu Zhang is currently a Bachelor student of Underground Structural Engineering of South China University of Technology. His research interests include data analysis and uncertainty quantification.

Cheng Su received his $\mathrm{PhD}$ in Civil Engineering from South China University of Technology, Guangzhou, China. He is currently a Full Professor of Civil Engineering Department of South China University of Technology. He is an editorial board member of Journal of Sound and Vibration. His research interests include computational mechanics, earthquake engineering, random vibration, and structural health monitoring.

\section{Introduction}

Bridge vehicular load effect inference is one of the key factors in bridge reliability and life-cycle assessment (Obrien et al., 2015), although many researches have been devoted to the load effect inference, most of them achieved the load effect based on over-simplified models of the vehicular load (Chen et al., 2010; Jiang, 2011). Inaccurate predictions on bridge load effects, especially for medium- or long-span bridges, might be achieved due to this over-simplification (Obrien et al., 2015).

The stochastic vehicular load can be decomposed into two components: the random vehicular inflow and the stochastic vehicular flow. The vehicular inflow component describes the information about the properties of the inflow vehicle entering the bridge, including the traffic volume, the vehicle weight and the vehicle entrance velocity. The traffic volume is a direct measure of the number of vehicles entering the bridge at different times. The vehicle weight includes the information about total vehicle weight, vehicle axle weights, vehicle axle lengths and inter-vehicle distances of each vehicle. The vehicle entrance velocity records the initial velocity of each vehicle entering the bridge. In practice, the above information can be measured if the bridge weigh-in-motion (WIM) sensing system is installed (Obrien et al., 2015). The vehicular flow component describes the information about the vehicle-following pattern of vehicles on the bridge. As it is difficult to directly measure the vehicular flow, the modelling of it relies on the simulation models. These models were developed to describe different aspects of traffic 
flow, and it can be categorised as microscopic, mesoscopic, and macroscopic modelling approaches (Hoogendoorn and Bovy, 2001):

1 the microscopic model considered the time-space behaviour of individual drivers under the influence of vehicles in their proximity

2 the mesoscopic model considered the behaviour of drivers without explicitly distinguishing their time-space behaviour

3 the macroscopic model considered the collective vehicular flow.

The microscopic model includes the safe-distance model (May, 1990), the stimulusresponse model (May, 1990; Leutzbach, 1988) and the particle pedestrian model (Hoogendoorn and Bovy, 2000a), etc. The mesoscopic model includes the multiclass gas-kinetic model (Hoogendoorn and Bovy, 2000b), the multiclass multilane model (Hoogendoorn, 1999) and the cluster models (Botma, 1978), etc. The macroscopic model includes the LWR model (Lighthill and Whitham, 1955), the Payne-type models (Payne, 1971, 1979) and the Helbing-type models (Helbing, 1996, 1997), etc. The cellular automaton (CA) or the particle hopping model was developed for modelling microscopic traffic flow, which was one of the popular methods for traffic flow simulation in recent years (Nagel and Schreckenberg, 1992). The most distinctive feature of this model is that it is discrete in time, space and states, thus, it is very suitable for simulation. The initial version of the CA model used for traffic flow simulation was the Nagel-Schreckenberg (N-S) model (Nagel and Schreckenberg, 1992), which was also one of the most important CA models. The N-S model can simulate different traffic scenarios in reality, such as congestion and phase transition. The N-S model has been utilised for the realistic description of stochastic vehicular flows, and it has been utilised in highway and bridge assessment, for instance, the transportation analysis and simulation system developed by Los Alamos National Laboratory (Smith et al., 1995) and the online traffic simulation in North Rhein Westphalia (Shafizadeh and Mannering, 2006). Further development of the $\mathrm{N}-\mathrm{S}$ model has been proposed for more complex situation of traffic flow, such as two-lane traffic flow based on different lane-changing methods (Kong et al., 2006; Caprani et al., 2016).

In the previous researches of using the N-S model to simulate the stochastic vehicular flow in bridge response analysis, it was found that the bridge load effects were related to the parameters of both the random vehicular inflow and the stochastic vehicular flow (Caprani et al., 2016; Yin et al., 2016; Mu et al., 2018). In order to investigate how the bridge load effect is affected by the stochastic vehicular load, two key parameters of the stochastic vehicular load are selected:

1 the probability of the existence of vehicle $(\mathrm{PoV})$, controlling the traffic volume of the vehicular inflow

2 the probability of random slowing down (PoSD), controlling the vehicle-following pattern of the stochastic vehicular flow.

With different values of the PoV and the PoSD, the samples of the vehicular load effects can be achieved by embedding the samples of the stochastic vehicular load and the influence lines of the load effects. In order to explore the relations between two parameters of the stochastic vehicular load (the PoV and the PoSD) and the statistical moments [the mean and the standard deviation (STD)], the Gaussian process regression 
(GPR) is utilised in this study. The GPR is a popular machine learning algorithm for regression (O'Hagan and Kingman, 1978; Mackay, 1997; Rasmussen, 2004; Chu and Ghahramani, 2005). Different from the traditional regression methods based on the polynomial functions of the input variables, the GPR models the data by the kernel functions of the input variable and makes prediction by the data-driven mean function and covariance function. Thus, it provides a more flexible solution for data fitting with reliable fitting capability in modelling complex systems. This powerful tool has been applied in the field of structural health monitoring (Surace et al., 2014; Corrado et al., 2015; Wan et al., 2017).

The structure of this paper is outlined as follows: Section 2 describes the simulation of the random vehicular inflow and the stochastic vehicular flow of the stochastic vehicular load model. Section 3 introduces the GPR for data fitting and prediction. Section 4 is the simulated example, with exploring the relations between the PoV along with the PoSD of the stochastic vehicular load and the mean and the STD of the load effect of a beam structure.

\section{Stochastic vehicular load: random vehicular inflow and stochastic vehicular flow}

Table 1 shows parameters of random vehicular inflow simulation. Recall that there are three factors governing the inflow, including the traffic volume, the vehicle weight and the vehicle entrance velocity. The traffic volume is described by the PoV, which is the direct measure of how probable of the existence of a vehicle in each time step. In order to investigate how the load effects are affected with changing the PoV, the values of the PoV are taken from 0.1 to 1.0 with 0.1 increasement. Note that PoV equal to 1.0 means that there is a vehicle existing at the entrance position of the bridge in each time step. As this study focuses on the influence of the traffic volume (i.e., the PoV) to the load effects, each vehicle is simplified as a point load with the identical weight (i.e., 10,000 N) and the entrance velocity (i.e., $0 \mathrm{~m} / \mathrm{s}$ ).

After generating the vehicles according to the vehicular inflow simulation, each vehicle moves according to the stochastic vehicular flow based on the N-S model. Discretisation in time and traffic lane is assumed. The time step is taken to be $1 \mathrm{sec}$ and the traffic lane of the bridge is divided into a set of cells with each cell-length being $2 \mathrm{~m}$. Each cell is either empty without any vehicle or occupied by a single vehicle. In each time step, each vehicle moves from one cell to another. Let $v_{i}(t), l_{i}(t)$ and $d_{i, i+1}(t)$ be the velocity, position, and inter-vehicle distance between the front vehicle (i.e., $(i+1)^{\text {th }}$ vehicle) and the target vehicle (i.e., $i^{\text {th }}$ vehicle), respectively. From time step $t$ to $t+1$, the vehicle-following pattern is modelled by four processes (acceleration, deceleration, random slowing down, position updating), so the position of the $i^{\text {th }}$ vehicle is updated as follows:

Table 1 Parameters of random vehicular inflow simulation

\begin{tabular}{lc}
\hline Parameter & Value \\
\hline PoV & $0.1: 0.1: 1.0$ \\
Total vehicle weight & $10,000 \mathrm{~N}$ \\
Vehicle entrance velocity & $0 \mathrm{~m} / \mathrm{s}$ \\
\hline
\end{tabular}


1 Acceleration

$$
v_{i}(t+1 / 3)=\min \left\{v_{i}(t)+v_{a c c}, v_{\max }\right\}
$$

2 Deceleration

$$
v_{i}(t+2 / 3)=\min \left\{v_{i}(t+1 / 3), d_{i, i+1}(t)\right\}
$$

3 Random slowing down with the PoSD

$$
v_{i}(t+1)=\max \left\{v_{i}(t+2 / 3)-v_{d e c}, 0\right\}
$$

$4 \quad$ Position updating

$$
l_{i}(t+1)=l_{i}(t)+v_{i}(t+1)
$$

Table 2 Parameters of the N-S model

\begin{tabular}{lc}
\hline Parameter & Value \\
\hline PoSD & $0.1: 0.1: 0.9$ \\
Maximum velocity limit & $30 \mathrm{~m} / \mathrm{s}$ \\
Velocity acceleration limit & $2 \mathrm{~m} / \mathrm{s}$ \\
Velocity deceleration limit & $2 \mathrm{~m} / \mathrm{s}$ \\
\hline
\end{tabular}

In the acceleration process, if the accelerated velocity of the $i^{\text {th }}$ vehicle is smaller than $v_{\max }$ (the maximum velocity limit), the velocity increases by $v_{a c c}$ (velocity acceleration limit). In the deceleration process, checking is performed on whether the updated position of the $i^{\text {th }}$ vehicle is in the same cell of its front vehicle (i.e., the $(i+1)^{\text {th }}$ vehicle). If no, then the position of the $i^{\text {th }}$ vehicle is updated; if yes, then the position of the $i^{\text {th }}$ vehicle is in the rear cell of the $(i+1)^{\text {th }}$ vehicle. In the random slowing down process, the velocity is decreased by $v_{d e c}$ (velocity deceleration limit) with the PoSD. Finally, the position of the $i^{\text {th }}$ vehicle is updated. Table 2 shows the parameters of the N-S model. In order to investigate how the load effects are affected with changing the PoSD, the values of the PoSD are taken from 0.1 to 0.9 with 0.1 increasement. Note that the PoSD directly controls how probable of a vehicle takes the slow down action of equation (3) in each simulation. As a result, the smoothness of the traffic state depends on both the PoSD and the PoV. The maximum velocity limit is $30 \mathrm{~m} / \mathrm{s}$ (i.e., $108 \mathrm{~km} / \mathrm{h}$ speed limit), the acceleration and deceleration limit are $2 \mathrm{~m} / \mathrm{s}$. By the above two components, the samples of the stochastic vehicular load samples can be achieved. Then, different load effects of the target structure can be calculated by the influence lines along with the vehicular load samples.

\section{Gaussian process regression}

Consider an input-output model:

$$
y=f(\boldsymbol{x})+\varepsilon
$$


where $x \in \mathbb{R}^{N_{x}}$ is the input; $y \in \mathbb{R}$ is the output; $\varepsilon \sim G\left(0, \sigma_{y}^{2}\right)$ is the noise following the zero-mean Gaussian distribution; and $f($.) is the predicting function. In the GRP, the covariance structure $\operatorname{cov}\left[y_{i}, y_{j}, \mid \boldsymbol{x}_{i}, \boldsymbol{x}_{j}, \theta_{\kappa}, \sigma_{y}^{2}\right]$ of two noisy outputs $y_{i}$ and $y_{j}$ is defined as (O’Hagan and Kingman, 1978; Mackay, 1997; Rasmussen, 2004; Chu and Ghahramani, 2005):

$$
\operatorname{cov}\left[y_{i}, y_{j} \mid \boldsymbol{x}_{i}, \boldsymbol{x}_{j}, \theta_{\kappa}, \sigma_{y}^{2}\right]=k\left(\boldsymbol{x}_{i}, \boldsymbol{x}_{j} \mid \theta_{\kappa}\right)+\sigma_{y}^{2} \delta\left(\boldsymbol{x}_{i}, \boldsymbol{x}_{j}\right)
$$

where $\boldsymbol{x}_{i}$ and $\boldsymbol{x}_{j}$ are the corresponding inputs of $y_{i}$ and $y_{j}$, respectively; $k\left(\boldsymbol{x}_{i}, \boldsymbol{x}_{j} \mid \theta_{\kappa}\right)$ is the kernel function conditional on the kernel parameter $\boldsymbol{\theta}_{\kappa}$, representing the correlation between the inputs $\boldsymbol{x}_{i}$ and $\boldsymbol{x}_{j} ; \delta\left(\boldsymbol{x}_{i}, \boldsymbol{x}_{j}=\mathbb{I}\left(\boldsymbol{x}_{i}=\boldsymbol{x}_{j}\right)\right.$ is the Kronecker delta. Based a training dataset $D=\{\mathbf{X}, \mathbf{Y}\}$ with $x \in \mathbb{R}^{N \times N_{x}}$ and $y \in \mathbb{R}^{N}$, and given a test input set $\mathrm{X} * \in$ $\mathbb{R}^{N * \times N_{x}}$, the corresponding probability density function (PDF) output set $y_{*} \in \mathbb{R}^{N_{*}}$ can be predicted by assuming that the joint follows the zero-mean $\left(N+N_{*}\right)$-dimensional multivariate Gaussian distribution (O’Hagan and Kingman, 1978; Mackay, 1997; Rasmussen, 2004; Chu and Ghahramani, 2005):

$$
\left[\begin{array}{c}
\mathbf{Y} \\
\mathbf{y}_{*}
\end{array}\right] \sim G\left(0,\left[\begin{array}{cc}
\mathbf{K}_{\mathbf{Y}} & \mathbf{K}_{*} \\
\mathbf{K}_{*}^{\mathbf{T}} & \mathbf{K}_{* *}
\end{array}\right]\right)
$$

where the covariance matrix of the training data $\mathbf{K}_{\mathbf{y}} \in \mathbb{R}^{N \times N}$ with its $(i, j)^{\text {th }}$ element as $k\left(\boldsymbol{x}_{i}, \boldsymbol{x}_{j}\right)+\sigma_{y}^{2} \delta\left(\boldsymbol{x}_{i}, \boldsymbol{x}_{j}\right)$; the covariance matrix between the training data and test data $\mathbf{K}_{*} \in \mathbb{R}^{N \times N *}$ with its $(i, j)^{\text {th }}$ element as $k\left(\boldsymbol{x}_{i}, \mathbf{x}_{*(j)}\right)+\sigma_{y}^{2} \delta\left(\boldsymbol{x}_{i}, \mathbf{x}_{*(j)}\right)$, where $\mathbf{x}_{*(j)}$ is the $j^{\text {th }}$ row of $x_{*}$; and the covariance matrix of the test data $\mathbf{K}_{* *} \in \mathbb{R}^{N * \times N_{*}}$ with its $(i, j)^{\text {th }}$ element as $k\left(\boldsymbol{x}_{*(i)}, \mathbf{x}_{*(j)}\right)+\sigma_{y}^{2} \delta\left(\boldsymbol{x}_{*(i)}, \mathbf{x}_{*(j)}\right)$. Finally, the posterior predictive density of $\mathbf{y}_{*}$ follows the $N_{*}$-dimensional Gaussian distribution as

$$
p\left(\mathbf{y} * \mid \mathbf{X}_{*}, \mathbf{X}, \mathbf{Y}\right) \sim G\left(\mathbf{K}_{*}^{\mathbf{T}} \mathbf{K}_{\mathbf{Y}}^{-1} \mathbf{Y}, \mathbf{K}_{* *}-\mathbf{K}_{*}^{\mathbf{T}} \mathbf{K}_{\mathbf{Y}}^{-1} \mathbf{K}_{*}\right)
$$

\section{Simulated example}

In this stimulated example, the stochastic vehicular load samples are achieved by considering the random vehicular inflow and stochastic vehicular flow components presented in Section 2. As the vehicular flow effect is more critical for medium- or long-span bridges, a 1,000-m simply supported beam is utilised in the study. Recall that the PoV and the PoSD vary according to Tables 1 and 2, respectively. For each PoV-PoSD parameter pair, for example, $\mathrm{PoV}=0.3$ and $\mathrm{PoSD}=0.8,10,000$ independent sets of samples of the vehicular load are generated, and each sample set is with 100 -sec time-span. The target load effect in this study is the maximum value of the mid-span moment within 100-sec time-span (denoted as max-mid-moment). In order to investigate how the time-space evolution of the stochastic vehicular load is affected by different values of the PoV and the PoSD, some typical samples of the vehicular time-space evolution are achieved as follows. First, the minimum, median, and maximum values of 
10,000 points of the max-mid-moment are achieved. Second, the vehicular time-space evolution samples correspond to the minimum, median, and maximum values of the max-mid-moment are selected for demonstration. Figures 1 to 3 show the time-space evolution of the stochastic vehicular load of the minimum, median, and maximum values of the max-mid-moment, respectively. The horizontal and vertical axes are time instance and position of the bridge, and each dot represents a vehicle being on the corresponding position of the bridge at the corresponding time instance. The vertical blue line indicates the time instance for the max-mid-moment. In Figure 1, it can be observed how the number of vehicles on the bridge (NoV) changes as varying the PoV and the PoSD. The NoV decreases as the PoSD increases. This is because when the PoSD is large, in every simulation each vehicle is with a high probability to decelerate as well as block its rear vehicle. It is not surprising that in the extreme case $(\operatorname{PoSD}=0.9)$, majority of vehicles stay at the entrance position of the bridge, i.e., the position of the vehicle is unchanged after updating. This is because the entrance velocity of each vehicle is equal to $0 \mathrm{~m} / \mathrm{s}$, and it is very probable that the effects of the first three processes of equations (1) to (3) cancel with each other. The NoV increases as the PoV increases. This is reasonable because larger value of the PoV leads to high traffic volume (i.e., number of vehicles at the bridge entrance). The above conclusions can also be found in Figures 2 and 3. A worth noting point is that traffic congestion can be observed in Figure 3. This validates the capability of the N-S model in modelling the smoothness of the traffic state.

Figure 1 Time-space evolution of the stochastic vehicular load of the minimum value of the max-mid-moment (see online version for colours)
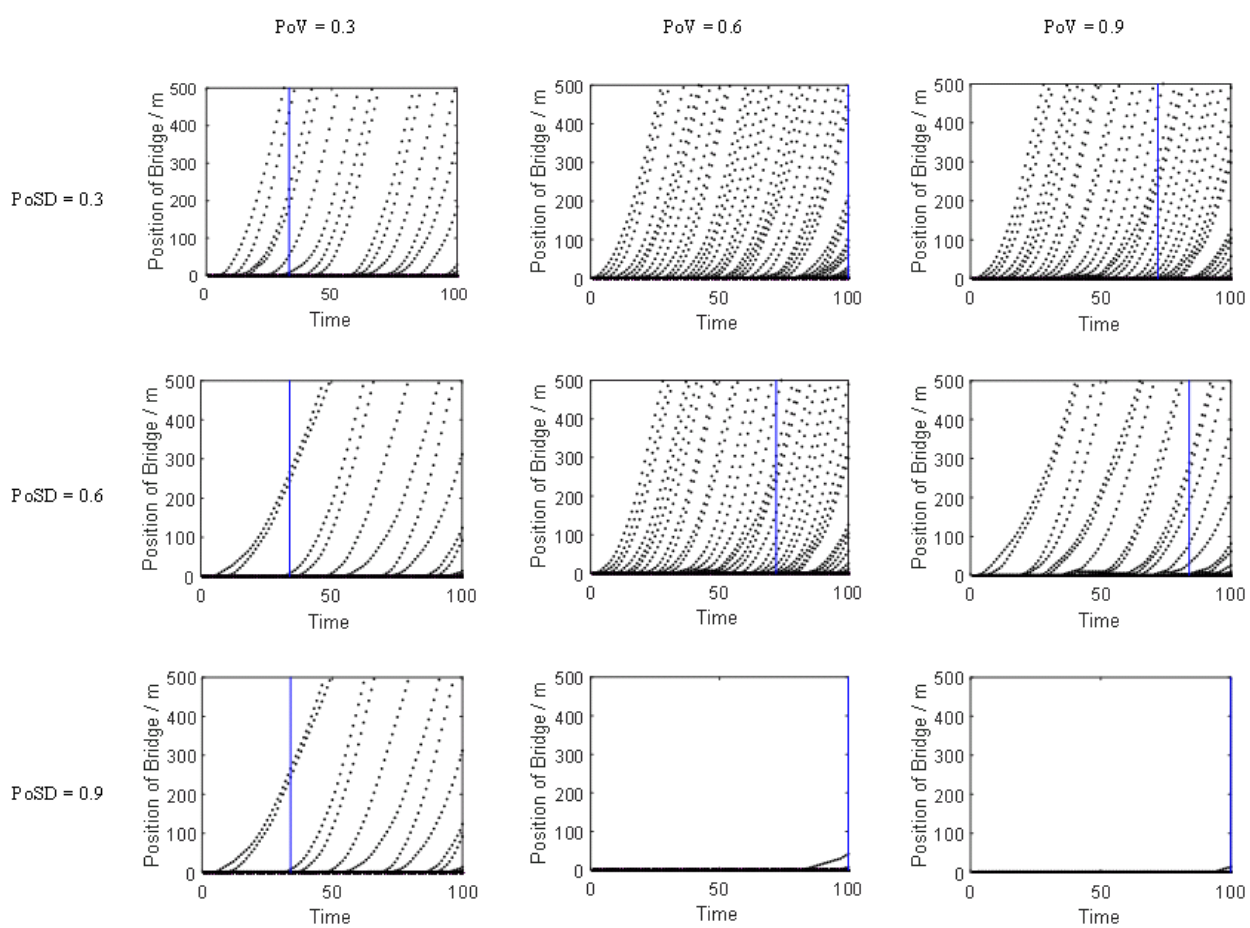
Figure 2 Time-space evolution of the stochastic vehicular load of the median value of the max-mid-moment (see online version for colours)

PoV $=0.3$
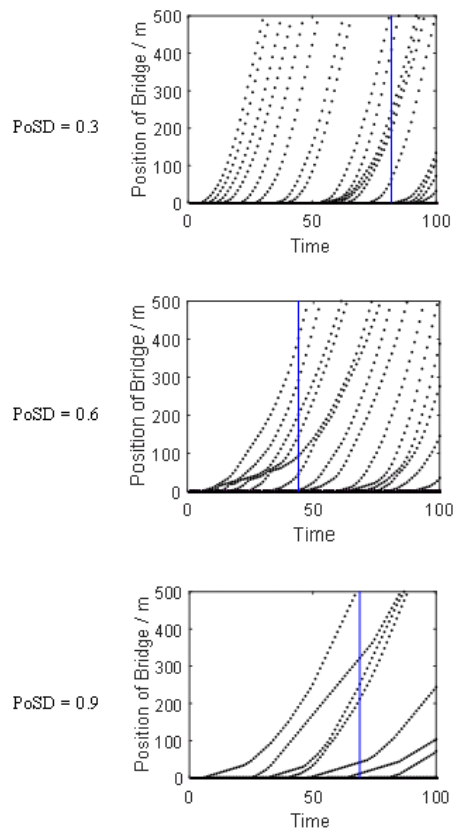

$\mathrm{PoV}=0.6$
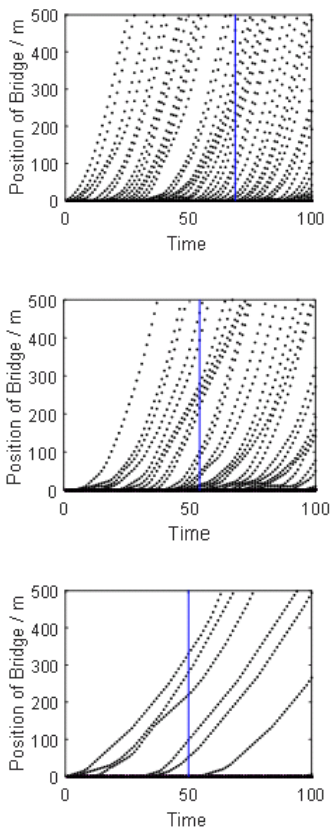

PoV $=0.9$
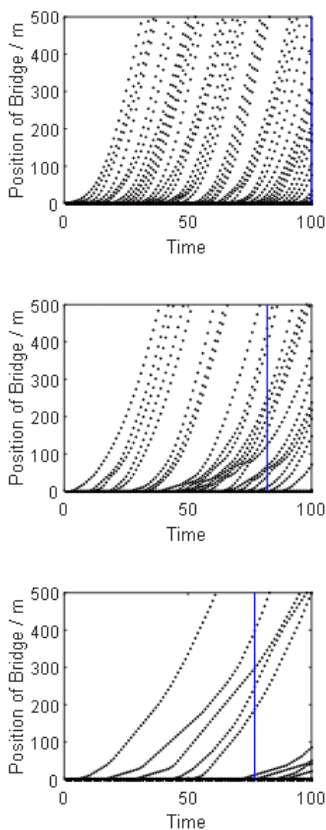

Figure 3 Time-space evolution of the stochastic vehicular load of the maximum value of the max-mid-moment (see online version for colours)

PoV $=0.3$
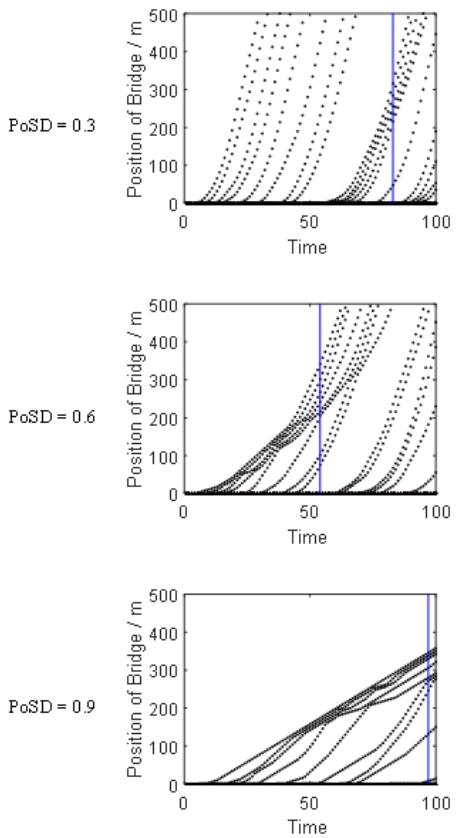

$\mathrm{PoV}=0.6$
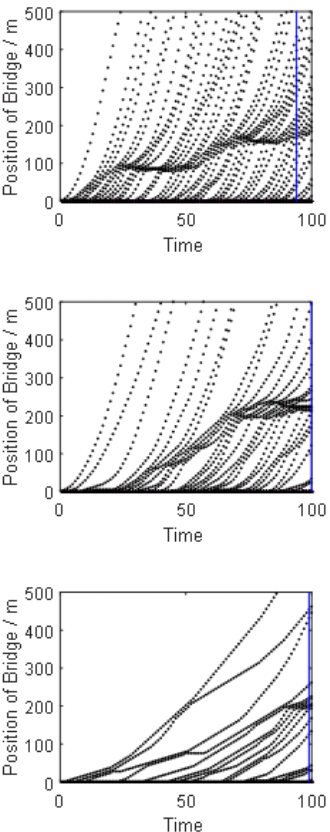

PoV $=0.9$
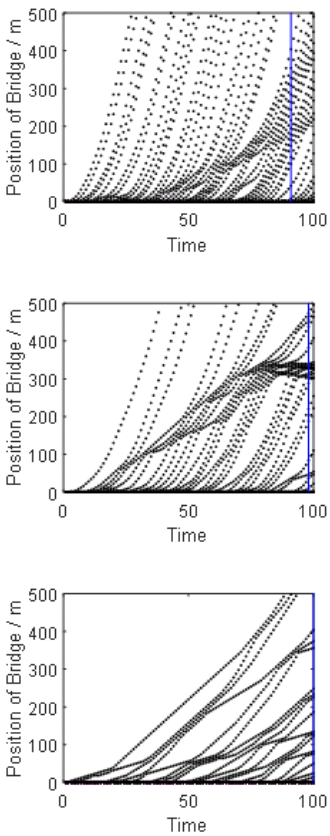
Figure 4 shows plot and normalised histograms of the max-mid-moment samples with fixing the PoV and varying the PoSD. It can be seen that the normalised histograms corresponding to different values of the PoV and the PoSD change significantly. When the PoV is fixed and the PoSD increases the lower bound of the histogram decreases while the upper bound of the histogram increases and then decreases. In addition, the shape of the normalised histograms derivates dramatically. Figure 5 shows plot and normalised histograms of the max-mid-moment samples with fixing the PoSD and varying the PoV. Again, changing on the normalised histograms can be observed, but the trends of the upper and lower bounds are more complex. Note that the max-mid-moment depends on not only the NoV but also the position of every vehicle on the bridge, and these two factors depend on the stochastic process parametrised by the PoV and PoSD.

Figure 4 Plot and normalised histograms of the max-mid-moment samples with fixing the PoV and varying the PoSD, (a) $\mathrm{PoV}=0.3$ with $\mathrm{PoSD}=0.1: 0.1: 0.9$ (b) $\mathrm{PoV}=0.6$ with $\mathrm{PoSD}=0.1: 0.1: 0.9$ (c) $\mathrm{PoV}=0.9$ with $\mathrm{PoSD}=0.1: 0.1: 0.9$ (see online version for colours)

Projection of samples

(a)

$\mathrm{PoV}=0.3$

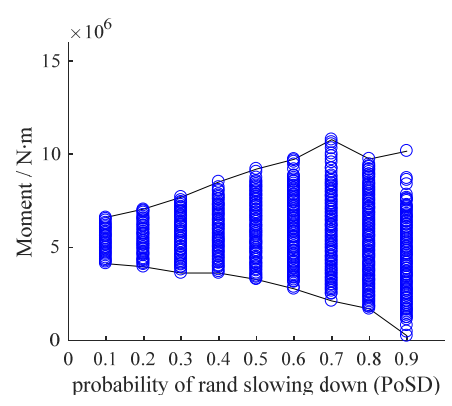

(b)

$\mathrm{PoV}=0.6$

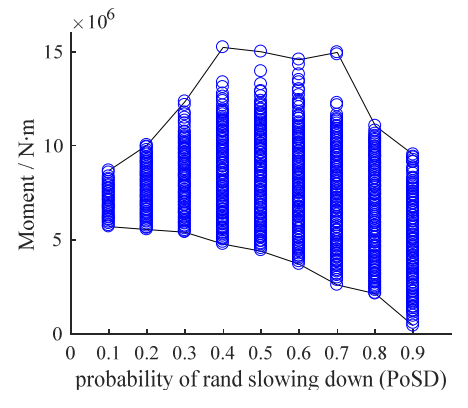

(c)

$\mathrm{PoV}=0.9$

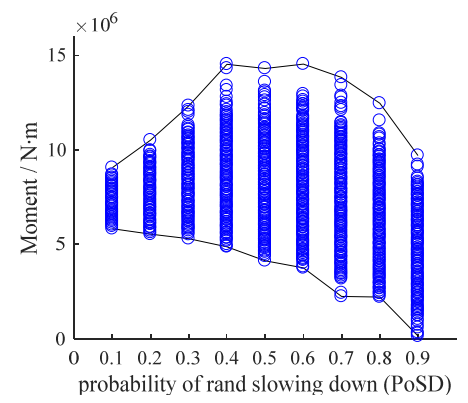

Normalised histogram of samples
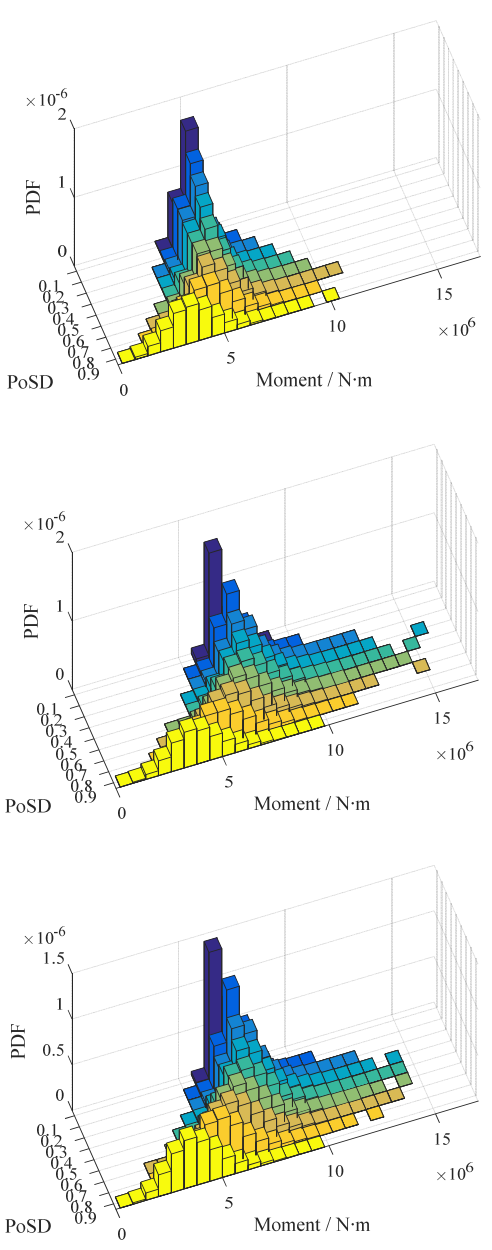
Figure 5 Plot and normalised histograms of the max-mid-moment samples with fixing the PoSD and varying the $\mathrm{PoV}$, (a) $\mathrm{PoSD}=0.3$ with $\mathrm{PoV}=0.1: 0.1: 1$ (b) $\mathrm{PoSD}=0.6$ with $\mathrm{PoV}=0.1: 0.1: 1$ (c) PoSD $=0.9$ with $\mathrm{PoV}=0.1: 0.1: 1$ (see online version for colours)

Plot of samples

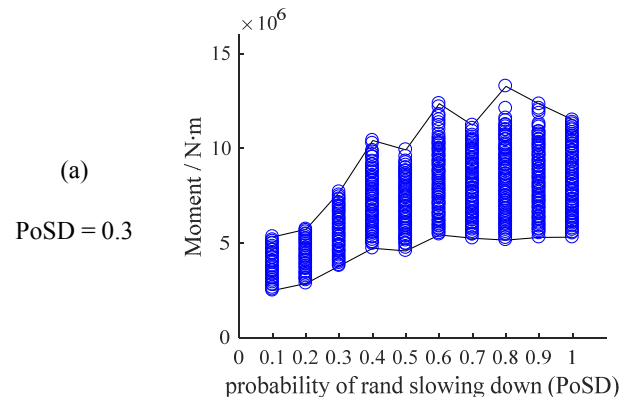

(b)

$\operatorname{PoSD}=0.6$

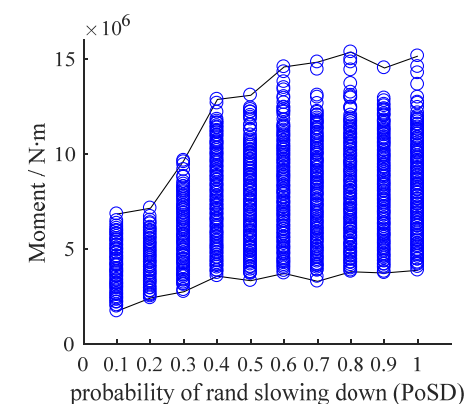

(c)

$\operatorname{PoSD}=0.9$

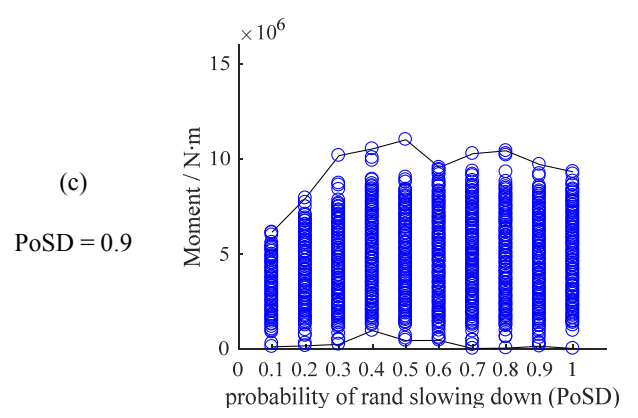

Normalised histogram of samples
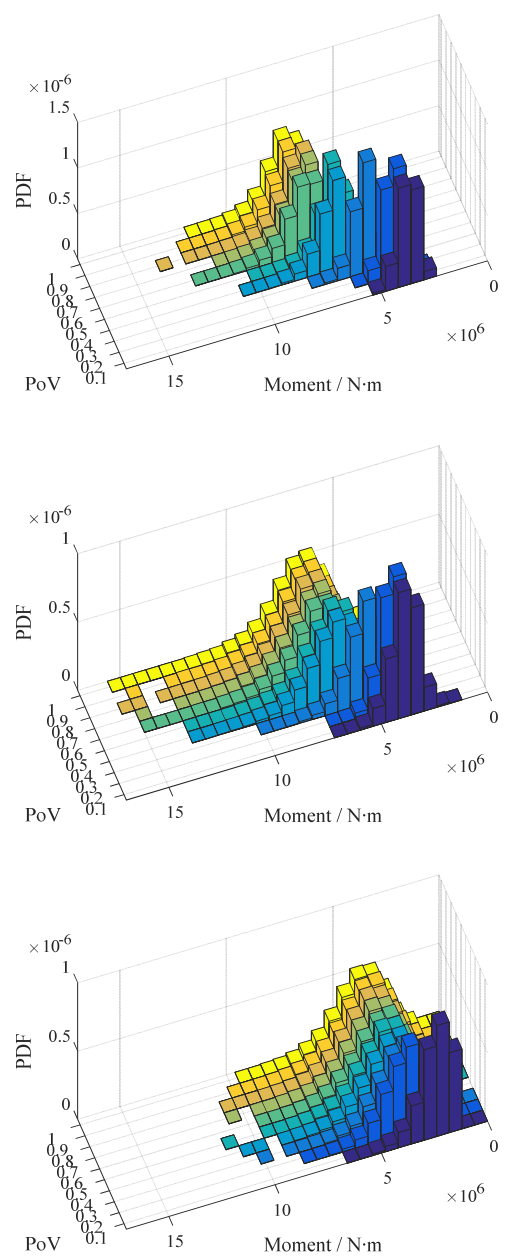

The GPR is utilised to analyse the above input-output dataset $D=\{\mathbf{X}, \mathbf{Y}\}$ with 10,000 points, containing the input dataset $\mathbf{X}$ : the PoV and the PoSD, and the output dataset $\mathbf{Y}$ : the max-mid-moment. In order to select the optimal kernel function and obtain the optimal values of the parameters, the mean squared error is utilised for the loss function. After the five-fold cross validation (Bishop, 2006), the squared exponential kernel function is selected for prediction. Figure 6 shows measured values versus GPR-based predicted values for the mean and STD of the max-mid-moment. It can be seen that the GPR gives good fitting accuracy. Figure 7 shows GPR-based prediction of the mean of the max-mid-moment. It can be observed that when the PoSD is fixed and the PoV increases, the mean of the max-mid-moment increases. When the PoSD increases, the increasing rate of the mean of the max-mid-moment becomes slower with increasing the 
PoV. When the PoSD reaches around 0.85 , the increasing rate of the mean of the maxmid-moment is very slow even though the PoV increases. When the PoV is fixed with being larger than around 0.4 and the PoSD increases, the mean of the max-mid-moment increases and then decreases. When the PoV is smaller than around 0.4, the changing rate of the mean of the max-mid-moment is slow even though the PoSD increases. Figure 8 shows GPR-based prediction of the mean of the max-mid-moment. It can be observed that when the PoSD is fixed and the PoV increases, the STD of the max-mid-moment increases. When the PoSD decreases, the increasing rate of the STD of the max-midmoment becomes slower with increasing the PoV. When the PoV is fixed with being larger than 0.45 and the PoSD increases, the STD of the max-mid-moment increases and then decreases. When the PoV is smaller than 0.45, the STD of the max-mid-moment increases as the PoSD increases. From the results shown in Figures 7 and 8, it turns out that the load effect is interactively influenced by the PoV and the PoSD:

1 The load effect depends on the NoV, the position of each vehicle on the bridge, and the smoothness of the traffic state.

2 The PoV controls the number of vehicles waiting at the entrance position of the bridge and the PoSD controls the number of vehicles driving onto the bridge. Larger PoV leads to larger NoV but large PoSD introduces traffic congestion at the bridge entrance and prohibits the vehicles driving onto the bridge.

3 The position updating of each vehicle on the bridge depends on the smoothness of the traffic state, and the smoothness depends on both the PoV and the PoSD. Larger $\mathrm{PoV}$ and PoSD lead to higher probability of the existence of traffic congestion. If traffic congestion happens, the vehicles are prohibited to leave the bridge, leading to larger NoV. As a result, the corresponding max-mid-moment is large.

Figure 6 Measured values versus GPR-based predicted values of the max-mid-moment, (a) mean (b) STD (see online version for colours)

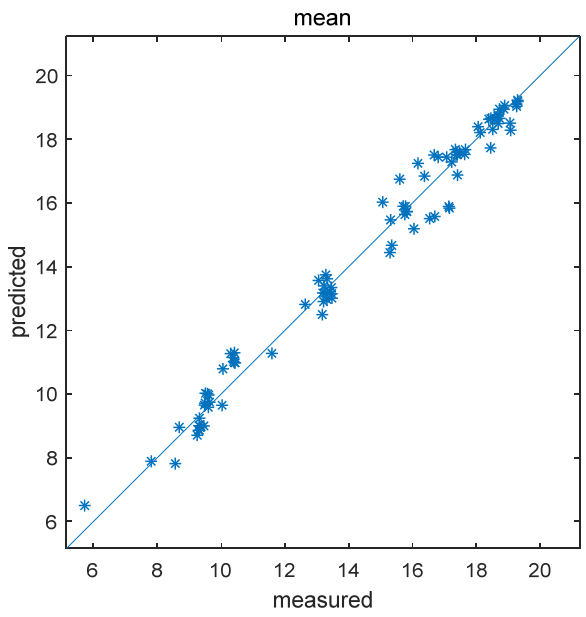

(a)

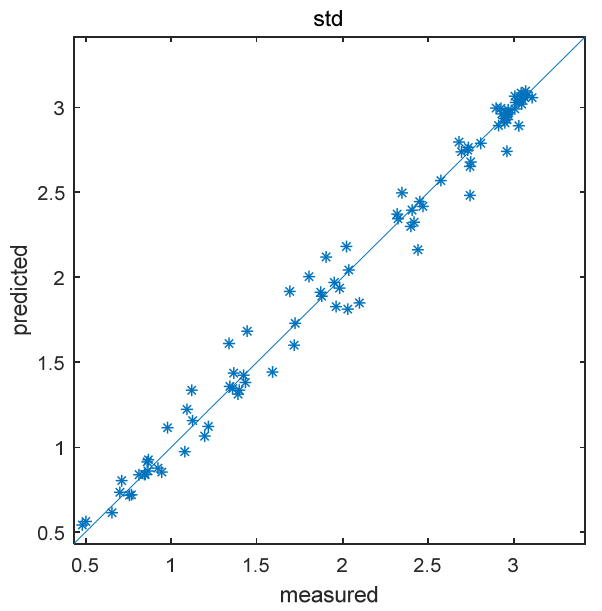

(b) 
Figure 7 GPR-based prediction of the mean of the max-mid-moment, (a) 3D surface (b) contour (see online version for colours)

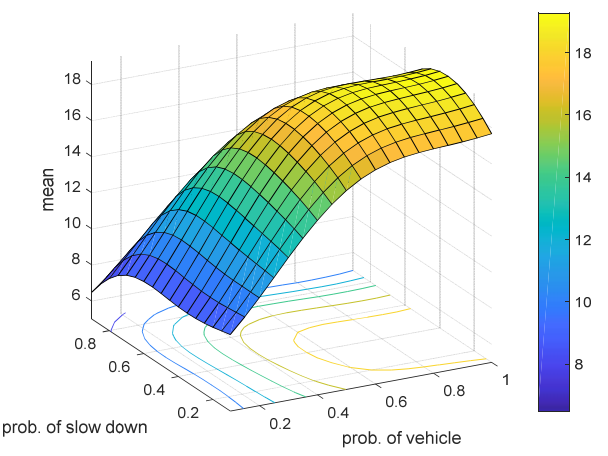

(a)

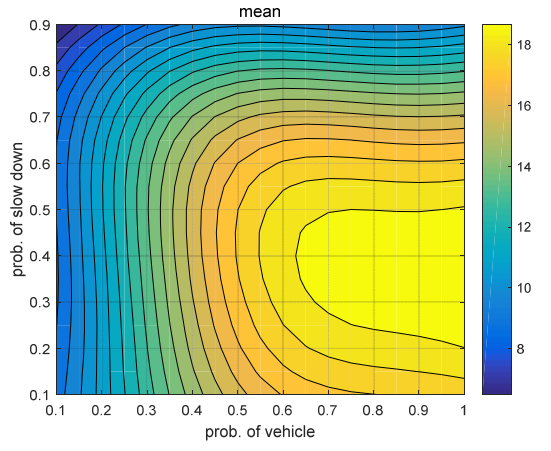

(b)

Figure 8 GPR-based prediction of the STD of the max-mid-moment, (a) 3D surface (b) contour (see online version for colours)
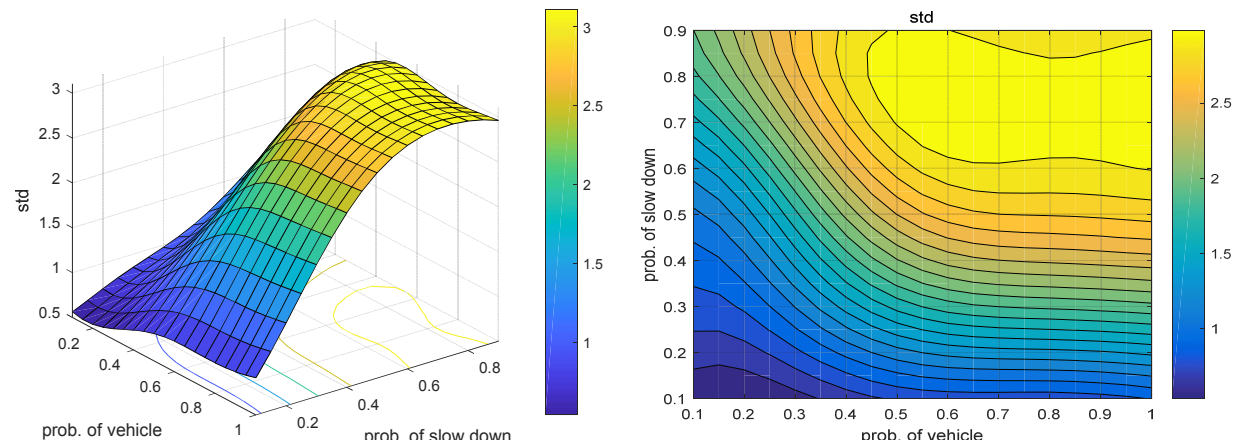

\section{Conclusions}

A parametric study of bridge load effect under stochastic vehicular load is conducted. The stochastic vehicular load is decomposed into the random vehicular inflow and the stochastic vehicular flow. Two key parameters (the PoV and the PoSD) of the stochastic vehicular load are selected. With different values of the PoV and the PoSD, the simulated samples of the vehicular load effects are achieved. A 1,000-m simply supported beam with the target load effect as the maximum value of the mid-span moment within 100-sec time-span is studied. The GPR, with good fitting capability, is utilised to obtain the relations between two parameters of the stochastic vehicular load (the PoV and the PoSD) and the statistical moments (the mean and the STD) of the simulated samples of the max-mid-moment. It turns out that the load effect depends on the NoV, the position of each vehicle on the bridge, and the smoothness of the traffic state. These factors are interactively influenced by the PoV and the PoSD. 


\section{Acknowledgements}

This work was supported by the National Natural Science Foundation of China (51508201, 51678252), the Natural Science Foundation of Guangdong Province, China (2017A030313262), Pearl River S\&T Nova Program of Guangzhou (201806010172), Science and Technology Program of Guangzhou (201804020069). This generous support is gratefully acknowledged.

\section{References}

Bishop, C.M. (2006) Pattern Recognition and Machine Learning, Springer, New York.

Botma, H. (1978) State-of-the-Art Report 'Traffic Flow Models', in Dutch, Research Report R-78-40, SWOV.

Caprani, C.C., Obrien, E.J. and Lipari, A. (2016) 'Long-span bridge traffic loading based on multi-lane traffic micro-simulation', Engineering Structures, Vol. 115, pp.207-219.

Chen, Y., Feng, M.Q. and Tan, C.A. (2010) 'Modeling of traffic excitation for system identification of bridge structures', Computer-Aided Civil and Infrastructure Engineering, Vol. 21, No. 1, pp.57-66.

Chu, W. and Ghahramani, Z. (2005) 'Gaussian processes for ordinal regression', Journal of Machine Learning Research, Vol. 6, No. 3, pp.1019-1041.

Corrado, N., Gherlone, M., Surace, C., Hensman, J. and Durrande, N. (2015) 'Damage localisation in delaminated composite plates using a Gaussian process approach', Meccanica, Vol. 50, No. 10, pp.2537-2546.

Helbing, D. (1996) 'Gas-kinetic derivation of navier-stokes-like traffic equations', Phys. Rev. E Stat. Phys. Plasmas. Fluids Relat. Interdiscip. Topics, Vol. 53, No. 3, pp.2366-2381.

Helbing, D. (1997) 'Modeling multi-lane traffic flow with queuing effects', Physica A, Vol. 242, Nos. 1-2, pp.175-194.

Hoogendoorn, S.P. (1999) Multiclass Continuum Modelling of Multiclass Traffic Flow, PhD thesis T99/5, TRAIL Thesis Series, Delft University Press.

Hoogendoorn, S.P. and Bovy, P. (2000a) 'Gas-kinetic modeling and simulation of pedestrian flows', Transportation Research Record Journal of the Transportation Research Board, Vol. 1710, No. 1, pp.28-36.

Hoogendoorn, S.P. and Bovy, P. (2000b) 'Modelling multiple user-class traffic flow', Transportation Research B, Vol. 34, No. 2, pp.123-146.

Hoogendoorn, S.P. and Bovy, P.H.L. (2001) 'State-of-the-art of vehicular traffic flow modelling', Proceedings of the Institution of Mechanical Engineers Part I Journal of Systems \& Control Engineering, Vol. 215, No. 4, pp.283-303.

Jiang, J.Q. (2011) 'Transient responses of timoshenko beams subject to a moving mass', Journal of Vibration \& Control, Vol. 17, No. 13, pp.1975-1982.

Kong, X.J., Gao, Z.Y. and Li, K.P. (2006) 'A two-lane cellular automata model with influence of next-nearest neighbor vehicle', Communications in Theoretical Physics, Vol. 45, No. 4, p.657.

Leutzbach, D.I.W. (1988) Introduction to the Theory of Traffic Flow, Springer, Berlin, Germany.

Lighthill, M.J. and Whitham, G.B. (1955) 'On kinematic waves: a theory of traffic flow on long crowded roads', Proceedings of the Royal Society A Mathematical Physical \& Engineering Sciences, Vol. 229, No. 1178, pp.317-345.

Mackay, D.J.C. (1997) 'Gaussian processes - a replacement for supervised neural networks?', Stochastic Modelling \& Applied Probability, Vol. 11, No. 3, pp.1-5. 
May, A.D. (1990) Traffic Flow Fundamentals, Prentice Hall, New Jersey, USA.

Mu, H.Q., Hu, Q., Guo, H.Z., Zhang, T.Y. and Su, C. (2018) 'Uncertainty quantification of load effects under stochastic traffic flows', International Journal of Structural Stability and Dynamics, DOI: 10.1142/S0219455419400091.

Nagel, K. and Schreckenberg, M. (1992) 'A cellular automaton model for freeway traffic', Journal De Physique I, Vol. 2, No. 12, pp.2221-2229.

O’Hagan, A. and Kingman, J.F.C. (1978) 'Curve fitting and optimal design for prediction', Journal of the Royal Statistical Society, Vol. 40, No. 1, pp.1-42.

Obrien, E.J., Schmidt, F., Hajializadeh, D., Zhou, X.Y., Enright, B., Caprani, C.C. et al. (2015) 'A review of probabilistic methods of assessment of load effects in bridges', Structural Safety, Vol. 53, pp.44-56.

Payne, H.J. (1971) 'Model of freeway traffic and control', Mathematical Models of Public Systems, Vol. 28, pp.51-61.

Payne, H.J. (1979) 'FREFLO: a macroscopic simulation model of freeway traffic', Transp. Res. Rec., No. 722, pp.68-77.

Rasmussen, C.E. (2004) 'Gaussian processes in machine learning', in Advanced Lectures on Machine Learning, pp.63-71, Springer, Berlin, Heidelberg.

Shafizadeh, K. and Mannering, F. (2006) 'Statistical modeling of user perceptions of infrastructure condition: application to the case of highway roughness', Journal of Transportation Engineering, Vol. 132, No. 2, pp.133-140.

Smith, L., Beckman, R., Anson, D., Nagel, K. and Williams, M. (1995) Transims: Transportation Analysis and Simulation System, Office of Scientific \& Technical Information Technical Reports.

Surace, C., Saxena, R., Gherlone, M. and Darwich, H. (2014) 'Damage localisation in plate like-structures using the two-dimensional polynomial annihilation edge detection method', Journal of Sound \& Vibration, Vol. 333, No. 21, pp.5412-5426.

Wan, H., Ren, W. and Todd, M.D. (2017) 'An efficient metamodeling approach for uncertainty quantification of complex systems with arbitrary parameter probability distributions', International Journal for Numerical Methods in Engineering, Vol. 109, No. 5, pp.739-760.

Yin, X., Yang, L., Guo, S., Zhang, W. and Cai, C.S. (2016) 'Three-dimensional vibrations of a suspension bridge under stochastic traffic flows and road roughness', International Journal of Structural Stability \& Dynamics, Vol. 16, No. 7, p.1550038. 